\title{
Finite Control Set Model Predictive Speed Control of a DC Motor
}

\author{
Viktor Šlapák, Karol Kyslan, Milan Lacko, Viliam Fedák, and František Ďurovský \\ Department of Electrical Engineering and Mechatronics, Faculty of Electrical Engineering and Informatics, \\ Technical University of Košice, Letná 9, 04200 Košice, Slovakia \\ Correspondence should be addressed to Karol Kyslan; karol.kyslan@tuke.sk
}

Received 10 February 2016; Revised 29 June 2016; Accepted 30 June 2016

Academic Editor: Zhen-Lai Han

Copyright (C) 2016 Viktor Šlapák et al. This is an open access article distributed under the Creative Commons Attribution License, which permits unrestricted use, distribution, and reproduction in any medium, provided the original work is properly cited.

\begin{abstract}
The paper describes the design procedure for a finite control set model predictive control (FCS-MPC) of brushed permanent magnet DC (PMDC) machine supplied from DC-DC converter. Full order linear Kalman filter is used for estimation of an unmeasured load torque and reduction of speed measurement noise. A new cost function has been introduced with a feedforward dynamic current component and a feedforward static load current component. The performance of the proposed control strategy is compared to the conventional PI-PWM cascade speed control through the experimental verification on the $250 \mathrm{~W}$ laboratory prototype. Obtained results show excellent dynamic behaviour and indicate possible energy savings of the proposed speed control.
\end{abstract}

\section{Introduction}

Permanent magnet DC (PMDC) machines with brushes are being of less importance today and they are continuously replaced by permanent magnet synchronous machines (PMSM) or brushless DC (BLDC) machines. Despite this fact, PMDC machines still have their field of use in various types of applications, from the machine tools and small home appliances to photovoltaic, robotic, and medical applications. They are widely used as auxiliary drives in automotive solutions due to presence of a DC grid in modern vehicles, and they are especially suitable for a battery operated equipment. The biggest drawback of these machines is a commutator, which needs regular maintenance and causes sparkles, and, therefore, the machines cannot be used in explosive environment. On the other hand, linear speed-torque curve, high torque at low speed, and the simplicity of torque/speed control are well-known advantages of the conventional PMDC machines. Coreless PMDC machines have almost no cogging torque, less vibrations, and smoother motor running at low speed against conventional PMDC machines.

In order to work in all four quadrants, these machines are often supplied by H-bridge DC-DC converter, which changes mean value of the voltage on the machine terminals. This converter uses pulse-width modulation (PWM) with 2-level or 3-level type of control.

The PMDC machines have been extensively studied and many advanced control strategies have been presented in the past, but they are studied by many researchers even today [1-7]. Among the new approaches, a new and attractive alternative for the control of $\mathrm{H}$-bridge supplied PMDC machine is finite control set model predictive control (FCSMPC) [8]. A framework for the analytical derivation of FCSMPC can be found in [9]. FCS-MPC has been applied to the control of several types of electrical drives. Techniques for reducing a steady-state error of FCS-MPC controlled $\mathrm{H}$-bridge have been presented in [10]. Detailed description of the algorithm design for the commutation torque ripple minimization applied to a BLDC machine can be found in [11]. Direct speed control of a PMSM for a single-mass system has been developed in [12]. Predictive strategy for the speed control of a two-mass system driven by PMSM has been proposed in [13], introducing reduced order extended Kalman filter for mitigation of oscillations and compensation of load torque disturbances. In authors' opinion and supported by the literature review, a FCS-MPC for the PMDC machine has not been reported yet. 
Novelty of this paper lies in the following: the predictive control strategy is, for the first time, proposed for the combination of DC-DC converter and PMDC machine. The proposed predictive control is compared to the conventional approach based on PI controllers. In addition, we have proposed a new cost function, which is different compared to the commonly used ones [9]. In the novel cost function, two current components are introduced: a current component covering a dynamic feedforward torque, $i_{F}$, and a current component covering a static load torque, $i_{L}$. We will also show how these components affect overall control performance of FCS-MPC controller.

\section{Drive Model}

2.1. Continuous Model. In this section, a continuous time state space model and discrete model for the PMDC machine are obtained. The stator dynamics of the PMDC machine is described by

$$
v_{a}=R_{a} i_{a}+L_{a} \frac{d i_{a}}{d t}+v_{\mathrm{emf}}
$$

where $R_{a}$ is the armature resistance, $L_{a}$ is the armature inductance, $i_{a}$ and $v_{a}$ are the armature current and voltage, respectively, and $v_{\mathrm{emf}}$ is the back-emf voltage. The electric torque generated by the motor is given by

$$
T_{e}=k_{T} i_{a}
$$

where $T_{e}$ is the magnitude of motor torque and $k_{T}$ is the motor torque constant. From (2) it is obvious that nonlinearity of magnetization curve is neglected in the machine model. The mechanical part of the machine is given by

$$
\frac{d \omega_{m}}{d t}=\frac{1}{J_{m}}\left(T_{e}-T_{l}-B \omega_{m}\right),
$$

where $J_{m}$ and $B$ are the motor inertia and viscous friction, respectively; $\omega_{m}$ is the shaft angular speed; and $T_{l}$ is the load torque acting as an unknown external disturbance. For the sake of simplicity, it is assumed that characteristic of the external torque is nearly constant and the sampling time is short enough. Then, the disturbance torque is independent to the motor state variables and can be included in the system model as an augmentative state variable [14]. The system equations are summarized in the state space representation as

$$
\begin{aligned}
\frac{d \mathbf{x}(t)}{d t} & =\mathbf{A}_{\mathbf{c}} \mathbf{x}(t)+\mathbf{b}_{\mathbf{c}} u(t), \\
\mathbf{y}(t) & =\mathbf{C}_{\mathbf{c}} \mathbf{x}(t),
\end{aligned}
$$

where

$$
\begin{aligned}
& \mathbf{x}=\left[\begin{array}{lll}
i_{a} & \omega_{m} & T_{l}
\end{array}\right]^{T}, \\
& \mathbf{y}=\left[\begin{array}{ll}
i_{a} & \omega_{m}
\end{array}\right]^{T}, \\
& u=v_{a},
\end{aligned}
$$

$$
\begin{aligned}
& \mathbf{A}_{\mathbf{c}}=\left[\begin{array}{ccc}
-\frac{R_{a}}{L_{a}} & -\frac{k_{T}}{L_{a}} & 0 \\
\frac{k_{T}}{J_{m}} & -\frac{B}{J_{m}} & -\frac{1}{J_{m}} \\
0 & 0 & 0
\end{array}\right], \\
& \mathbf{b}_{\mathbf{c}}=\left[\begin{array}{c}
\frac{1}{L_{a}} \\
0 \\
0
\end{array}\right], \\
& \mathbf{C}_{\mathbf{c}}=\left[\begin{array}{lll}
1 & 0 & 0 \\
0 & 1 & 0
\end{array}\right] .
\end{aligned}
$$

2.2. Sampled-Data Model. The continuous model is approximated by a sampled-data model, which is needed in FCSMPC. Very simple strategy on how to obtain a discrete model is Euler forward or backward approximation. But in [15], it was shown that truncated Taylor series is more accurate and ensures no additional delays in the sampleddata model. Furthermore, it enables direct feedthrough from the actuation input to all states, which is necessary for the predictive control. An approximate model was obtained from the truncation of the Taylor series for sampling time, $T_{s}$, for each of the state components $x_{j}$ as follows:

$$
x_{j}(k+1)=x_{j}(k)+\left.\sum_{l=1}^{N_{j}} \frac{T_{s}^{l}}{l !} \frac{d^{l} x_{j}}{d t^{l}}\right|_{t_{k}} .
$$

The truncation should be performed until the input explicitly appears in the expansion [15]. For the current equation (1), the order used is $N_{j}=1$, but, for the angular speed equation (3), $N_{j}=2$. It results in the following sampled-data model:

$$
\begin{aligned}
\mathbf{x}(k+1) & =\mathbf{A}_{\mathbf{d}} \mathbf{x}(k)+\mathbf{b}_{\mathbf{d}} u(k), \\
\mathbf{y}(k) & =\mathbf{C}_{\mathbf{d}} \mathbf{x}(k),
\end{aligned}
$$

where

$$
\begin{aligned}
& \mathbf{b}_{\mathbf{d}}=\left[\begin{array}{l}
k_{3} \\
k_{7} \\
0
\end{array}\right], \\
& \mathbf{C}_{\mathbf{d}}=\left[\begin{array}{lll}
1 & 0 & 0 \\
0 & 1 & 0
\end{array}\right], \\
& k_{1}=1-\frac{T_{s} R_{a}}{L_{a}}, \\
& k_{2}=\frac{T_{s} k_{T}}{L_{a}},
\end{aligned}
$$$$
\mathbf{A}_{\mathbf{d}}=\left[\begin{array}{ccc}
k_{1} & -k_{2} & 0 \\
-k_{4} & k_{5} & k_{6} \\
0 & 0 & 1
\end{array}\right]
$$ 


$$
\begin{aligned}
& k_{3}=\frac{T_{s}}{L_{a}}, \\
& k_{4}=\frac{T_{s} k_{T}\left(B L_{a} T_{s}+J_{m} R_{a} T_{s}-2 J_{m} L_{a}\right)}{2 L_{a} J_{m}^{2}}, \\
& k_{5}=\frac{B^{2} L_{a} T_{S}^{2}-J_{m} T_{S}^{2} k_{T}^{2}-2 B J_{m} L_{a} T_{s}+2 L_{a} J_{m}^{2}}{2 L_{a} J_{m}^{2}}, \\
& k_{6}=\frac{T_{s}\left(B T_{s}-2 J_{m}\right)}{2 J_{m}^{2}}, \\
& k_{7}=\frac{k_{T} T_{s}^{2}}{2 J_{m} L_{a}} .
\end{aligned}
$$

\section{State Observer}

In this section, we introduce a full order Kalman filter to estimate the slowly varying load torque and to reduce impact of a quantization noise on the measurement of angular speed. $\mathrm{KF}$ proposed here is based on the predictor-corrector form [16]. It is assumed that state variables are excited by random disturbances (e.g., inaccurately modeled dynamics), and so we consider the sampled-data model (7) and (8) in the presence of noise as follows:

$$
\begin{aligned}
\mathbf{x}(k+1) & =\mathbf{A}_{\mathbf{d}} x(k)+\mathbf{B}_{\mathbf{d}} u(k)+\mathbf{v}(k), \\
\mathbf{y}(k) & =\left[\begin{array}{c}
\omega_{m}(k) \\
i_{a}(k)
\end{array}\right]+\mathbf{w}(k),
\end{aligned}
$$

where $\mathbf{v}(k)$ is the vector of zero-mean process noise and $\mathbf{w}(k)$ is the vector of zero-mean measurement noise. These noises are assumed uncorrelated as follows:

$$
\begin{aligned}
& \mathbf{v}(k)=\left[\begin{array}{l}
v_{1}(k) \\
v_{2}(k) \\
v_{3}(k)
\end{array}\right], \\
& \mathbf{w}(k)=\left[\begin{array}{l}
w_{1}(k) \\
w_{2}(k)
\end{array}\right] .
\end{aligned}
$$

Process noise auto-covariance matrix, $\mathbf{Q}$, is assumed to be

$$
\mathbf{Q}=\operatorname{diag}\left[q_{11}, q_{22}, q_{33}\right],
$$

where $q_{11}$ is the variance of $v_{1}(k)$ and so forth. Measurement noise auto-covariance matrix, $\mathbf{R}$, is assumed to be

$$
\mathbf{R}=\operatorname{diag}\left[r_{11}, r_{22}\right],
$$

where $r_{11}$ is the variance of $w_{1}(k)$ and so forth.

Then, the state estimate of the machine, which is further used in the control system (e.g., posteriori estimate), is given by

$$
\mathbf{x}_{\mathbf{c}}(k)=\mathbf{x}_{\mathbf{p}}(k)+\mathbf{K}\left[y(k)-y_{p}(k)\right] .
$$

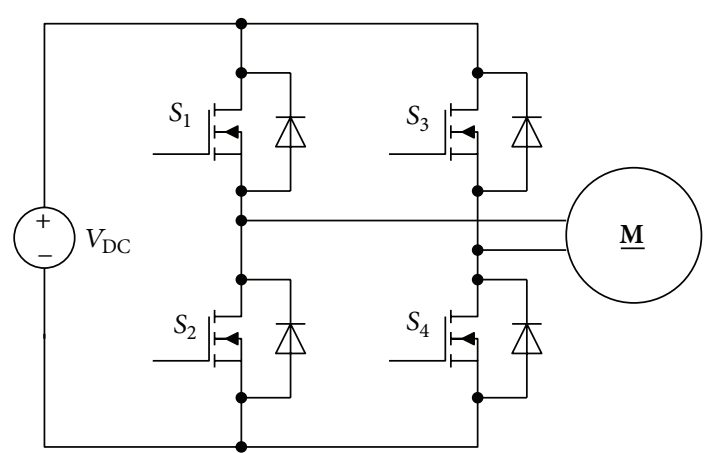

Figure 1: H-bridge DC-DC converter.

$\mathbf{K}$ is the Kalman gain given by

$$
\begin{aligned}
\mathbf{K}(k) & =\mathbf{P}_{\mathbf{p}}(k) \mathbf{C}_{\mathbf{d}}^{\mathrm{T}}\left[\mathbf{C}_{\mathbf{d}} \mathbf{P}_{\mathbf{p}}(k) \mathbf{C}_{\mathbf{d}}^{\mathrm{T}}+\mathbf{R}\right]^{-1}, \\
\mathbf{P}_{\mathbf{c}}(k) & =\left[\mathbf{I}-\mathbf{K}(k) \mathbf{C}_{\mathbf{d}}\right] \mathbf{P}_{\mathbf{p}}(k), \\
\mathbf{P}_{\mathbf{p}}(k+1) & =\mathbf{A}_{\mathbf{d}} \mathbf{P}_{\mathbf{c}}(k) \mathbf{A}_{\mathbf{d}}^{\mathrm{T}}+\mathbf{Q} .
\end{aligned}
$$

Predicted state estimate (e.g., a priori estimate) is given by

$$
\begin{aligned}
\mathbf{x}_{\mathbf{p}}(k+1) & =\mathbf{A}_{\mathbf{d}} x_{p}(k)+\mathbf{B}_{\mathbf{d}} u(k) . \\
\mathbf{y}_{p}(k) & =\mathbf{C}_{\mathbf{d}} x_{p}(k),
\end{aligned}
$$

The model (7) and (8) is assumed to be linear and time invariant. Thus, Kalman filter gain, $\mathbf{K}$, will converge towards a steady-state gain. We precalculated value of the gain matrix, $\mathbf{K}$, offline and then we use it in the online predictive control strategy. It is obvious that measurement noise is introduced by the incremental encoder and the current sensor. The incremental encoder with $N$ pulses per revolution (ppr) is evaluated within the sampling time, $T_{\mathrm{ENC}}$. With quadrature evaluation in the control system, it yields to the quantization error that

$$
\Delta \omega_{m}=\frac{2 \pi}{4 N T_{\mathrm{ENC}}} .
$$

Regarding a standard deviation, $\sigma_{22}$, of

$$
\sigma_{22}=\frac{1}{2} \Delta \omega_{m}
$$

and for $T_{\mathrm{ENC}}=500 \mu \mathrm{s}$ and $N=1024 \mathrm{ppr}$, the variance of the speed measurement noise is then obtained by

$$
r_{22}=\sigma_{22}^{2}=2.46 \text {. }
$$

\section{Power Converter}

PMDC motor is driven by conventional H-bridge (Figure 1) fed by DC link with the voltage value $V_{\mathrm{DC}}$. The $\mathrm{H}$-bridge has four admissible switching states, generating three output voltage values, $+V_{\mathrm{DC}},-V_{\mathrm{DC}}$, and zero voltage (Table 1 ).

In fact, there are two possibilities of PWM control of such DC-DC converter: two-level or three-level voltage control. 
TABLE 1: Admissible switching combinations.

\begin{tabular}{lc}
\hline$S_{1}, S_{4}$ & $+V_{\mathrm{DC}}$ \\
$S_{3}, S_{2}$ & $-V_{\mathrm{DC}}$ \\
$S_{1}, S_{3}$ & 0 \\
$S_{2}, S_{4}$ & 0 \\
\hline
\end{tabular}

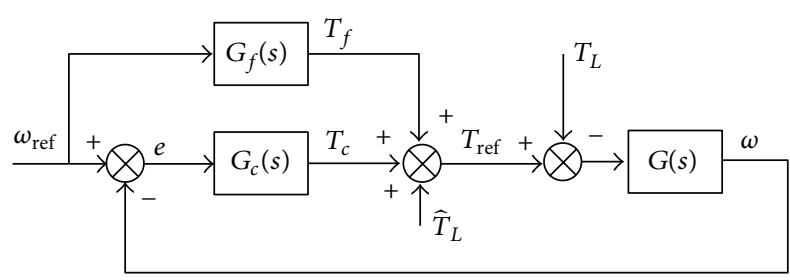

FIGURE 2: Classical speed control with 2DOF structure.

In the first case, both legs of converter are complementary controlled, and thus only positive or negative DC bus voltage is applied. In the second case, one leg holds defined switching state and the other leg is switching according to voltage demand. FCS-MPC does not use exactly one or another approach. Instead, the switching state is directly chosen by a FCS-MPC controller, as explained in Section 6.

\section{Speed Control Structure with PI Controller and Feedforward Compensation}

In this section, we provide only a short note of the conventional speed control based on PI controller, shown in Figure 2. It is presented here for two reasons: we will compare it to the novel predictive controller and, furthermore, it creates a basic theory to introduction of novel current components. Later, these components will be implemented into a cost function.

It is expected that torque response of PMDC machine is several times faster than the closed loop mechanical dynamics. Thus, the electrical dynamics can be neglected $\left(T_{e}=T_{\text {ref }}\right)$, and torque setpoint, $T_{\text {ref }}$, can be described by the following:

$$
T_{\text {ref }}=T_{c}+T_{f}+\widehat{T}_{L},
$$

where $T_{c}$ is the output of the PI controller, $T_{f}$ is the dynamic feedforward torque, and $\widehat{T}_{L}$ is the feedforward compensation of estimated load torque. With the use of the standard PI controller having transfer function,

$$
G_{c}(s)=K_{p}+\frac{K_{i}}{s},
$$

and simple mechanical dynamics (neglecting often a very small value of $B$ ),

$$
G(s)=\frac{1}{s J_{m}},
$$

the closed loop transfer function of the system in Figure 2 without feedforward (i.e., at $G_{f}(s)=0$ ) will be

$$
\frac{\omega(s)}{\omega_{\text {ref }}(s)}=\frac{s\left(K_{p} / J_{m}\right)+K_{i} / J_{m}}{s^{2}+\left(K_{p} / J_{m}\right) s+K_{i} / J_{m}} .
$$

In servo applications, when the system is driven from one position to another, trapezoidal speed references are used instead of stepwise speed change. Speed reference during acceleration and deceleration must be tracked without any steady-state error [17]. This can be achieved with two-degreeof-freedom (2DOF) control structure. In other words, the feedforward $G_{f}(s)$ must be properly set up. It can be easily shown that if the feedforward term is used in Figure 2,

$$
G_{f}(s)=s J_{m},
$$

closed loop transfer function (22) changes to an ideal unity gain. Because we neglected the dynamics of the electrical circuit and parameters of the machine may vary with time, the unity gain is only an ideal case. The control structure in Figure 2 with the feedforward is capable of assuring a tracking of trapezoidal speed reference with the high precision.

It is obvious from (23) that if the feedforward is to be applied, a value of reference angular acceleration, $\varepsilon_{\text {ref }}$, must be available. In servo applications, this value is generated from a trajectory generator or a ramp generator [18]. Based on (2) and (23), we can write an equation for a dynamic feedforward component of current as follows:

$$
i_{F}=\frac{1}{k_{T}} J_{m} \varepsilon_{\text {ref }} .
$$

In a similar way, a static load torque component of current can be written as

$$
i_{L}=\frac{1}{k_{T}} \widehat{T}_{L}
$$

We formulated these current components for later use in the novel cost function and we will also show their influence on the control performance.

\section{Novel FCS-MPC Based Control Structure}

6.1. Control Algorithm. Block diagram of the FCS-MPC speed control is shown in Figure 3. The proposed algorithm uses predictions of the future machine state in $(k+1)$ step for each value of admissible voltage. It means that three predictions are needed in each cycle. The prediction itself is based on the modified sampled-data model described in Section 2:

$$
\begin{aligned}
x_{p i}(k+1)= & {\left[\begin{array}{cc}
k_{1} & -k_{2} \\
-k_{4} & k_{5}
\end{array}\right]\left[\begin{array}{c}
\hat{i}_{a}(k) \\
\widehat{\omega}_{m}(k)
\end{array}\right] } \\
& +\left[\begin{array}{cc}
k_{3} & 0 \\
k_{7} & k_{6}
\end{array}\right]\left[\begin{array}{c}
u_{i}(k) \\
\widehat{T}_{L}(k)
\end{array}\right],
\end{aligned}
$$

where $\hat{i}_{a}$ and $\widehat{\omega}_{m}$ are the current and the speed filtered by $\mathrm{KF}, \widehat{T}_{L}$ is the estimated load torque, considered as the second system input introduced in order to predict correctly the future states, $x_{p i}$ is the vector of predicted states for $i$ th admissible voltage, and

$$
u_{i}(k) \in\left\{+V_{\mathrm{DC}}, 0,-V_{\mathrm{DC}}\right\}
$$




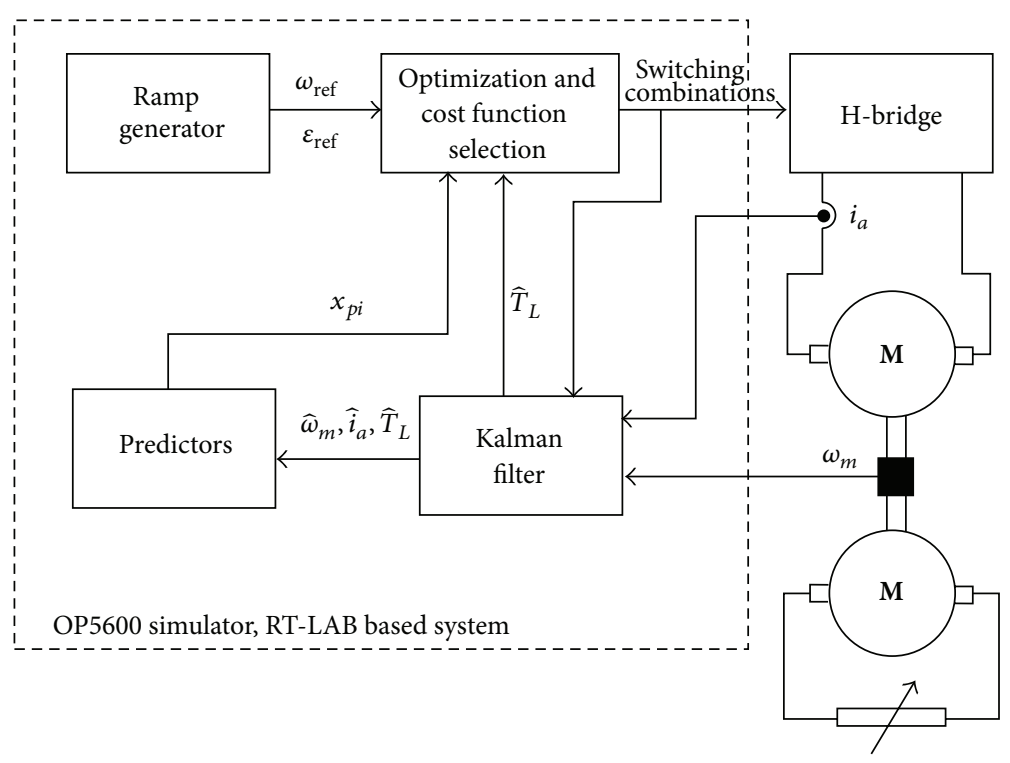

FIGURE 3: Block diagram of FCS-MPC control.

is the admissible voltage value. The predicted values are compared using cost function and the voltage, for which the cost function is evaluated as minimal, is then applied to the converter. Figure 4 presents the control algorithm for FCSMPC of H-bridge driven PMDC machine.

It consists of steps that can be summarized as follows:

(1) Measurement of the machine current and speed.

(2) Filtration of the current and speed by KF and estimation of a load torque.

(3) Calculation of required current components $i_{F}$ and $i_{L}$.

(4) Prediction of the future values of speed and current for each admissible voltage.

(5) Evaluation of the cost function, $J$, for three voltages, using variable state as an argument for which the cost function $J$ is evaluated as minimal (compare to [17]).

(6) Application of the optimal actuation to the H-bridge.

6.2. Controller Design. The following criteria have been proposed for the predictive controller:

(i) Precise reference tracking for the step and trapezoidal speed reference.

(ii) Limitation of the maximal current.

(iii) Smooth behaviour of the electrical torque.

(iv) Fast disturbance rejection.

The cost function implementation itself can be considered as a controller, which can fulfill abovementioned demands at once. The following cost function has been introduced:

$$
\begin{aligned}
J= & \underbrace{\lambda_{1}\left(\omega_{\text {ref }}-\omega_{m p}\right)^{2}}_{a}+\underbrace{\lambda_{2}\left(i_{F}+i_{L}-i_{a p}\right)^{2}}_{b} \\
& +\underbrace{f_{\lim }\left(i_{a p}\right)}_{c},
\end{aligned}
$$

where $\omega_{m p}$ and $i_{a p}$ are predicted values of the machine angular speed and current, respectively. The cost function consists of the sum of three terms:

(a) This term favors the voltage vectors that achieve reference speed tracking and it is weighted with weighting factor, $\lambda_{1}$.

(b) This term provides the current control of the machine and is weighted with weighting factor, $\lambda_{2}$. The summation of the aforementioned currents $i_{F}$ and $i_{L}$ is the current reference.

(c) This term in the cost function presents current limitation, where $f_{\text {lim }}$ is nonlinear function defined as in [17]:

$$
f_{\lim }\left(i_{a p}\right)= \begin{cases}\infty & \text { if }\left|i_{a p}\right|>i_{\mathrm{MAX}} \\ 0 & \text { if }\left|i_{a p}\right|<i_{\mathrm{MAX}}\end{cases}
$$

The current limitation works on the simple principle that when predicted value of the current for any voltage is above the limit, $i_{\mathrm{MAX}}$, the cost function gets very high value $\left(10^{12}\right)$, forcing the controller to choose another voltage.

\section{Experimental Results}

Proposed control algorithm was experimentally verified on a laboratory PMDC motor supplied by the H-bridge converter prototype, which was properly modified to receive external gate signals. The machine was equipped with optical incremental sensor LARM having the resolution of $1024 \mathrm{ppr}$. Parameters of the machine can be found in Appendix. Realtime HIL simulator OP5600, from Opal RT, was used as control hardware and algorithms were executed with the sampling time $50 \mu \mathrm{s}$. Control algorithm was developed in Matlab and modified on RT-LAB software in order to be 


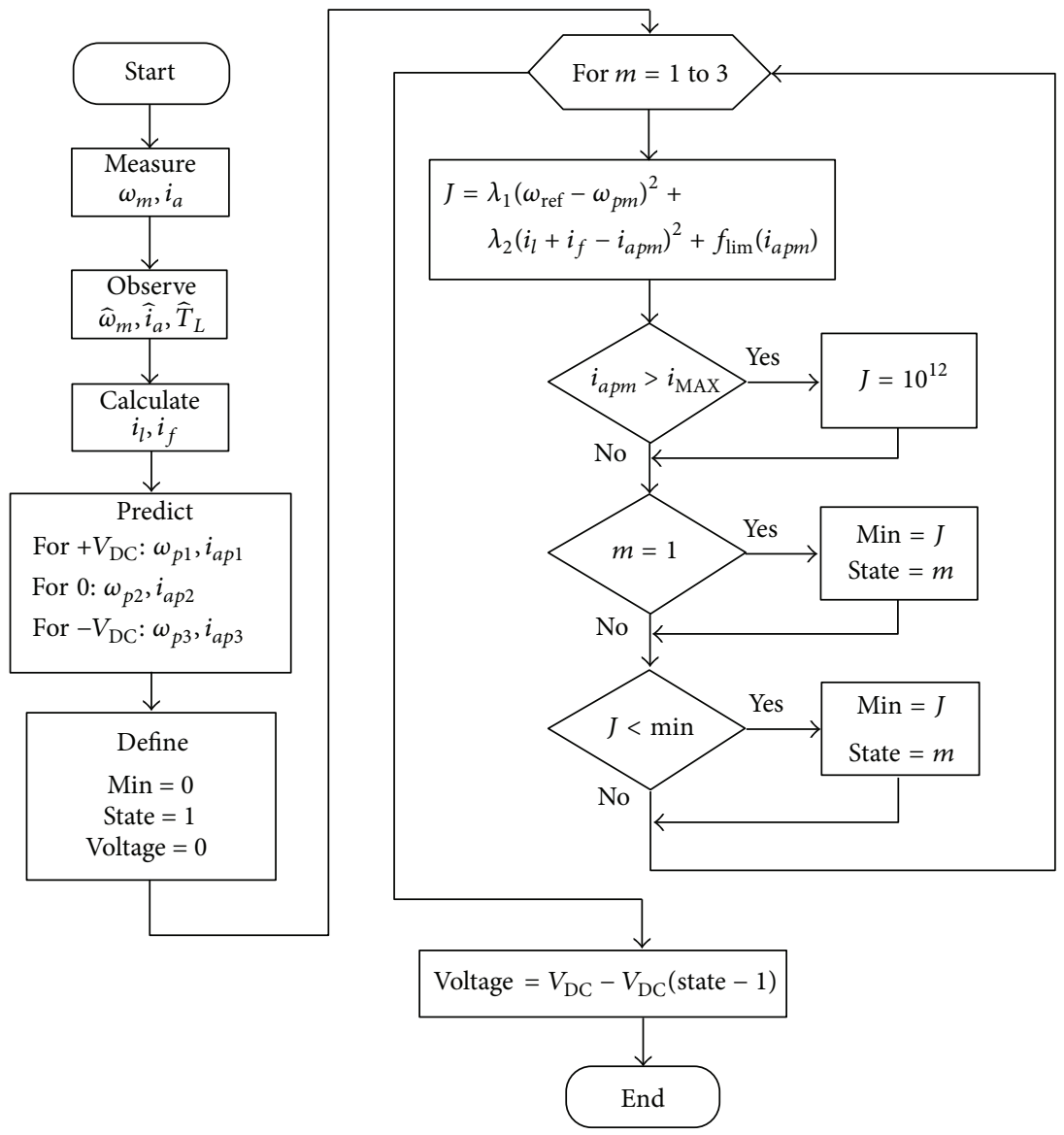

FIGURE 4: Flowchart of proposed FCS-MPC control algorithm.

implemented into simulator. The second PMDC machine was used as a load machine with the same parameters. Experimental results were taken for both control approaches under the same conditions, such as the current value limitation $i_{\mathrm{MAX}}=10 \mathrm{~A}$ and the sampling time $T_{s}=50 \mu \mathrm{s}$.

A standard cascade speed control structure with the inner current control loop and the superimposed speed control loop with antiwindup and dynamic feedforward is denoted in experimental results as PI. It uses 2-level voltage control with $10 \mathrm{kHz}$ PWM frequency. FCS-MPC speed control, as presented in Section 6, is denoted in experimental results as MPC. For a good comparability of both methods, the controllers were tuned to reach the same settling time and overshoot. It resulted in the values $K_{p}=0.5 \mathrm{Nms}$ and $K_{i}=$ $11 \mathrm{Nm}$ for PI controller and $\lambda_{1}=150$ and $\lambda_{2}=1$ for MPC controller.

The experimental results comparing these control approaches are shown in Figures 5-8. Figure 5 shows the results of a reference step from 0 to $80 \mathrm{rad} / \mathrm{s}$. Looking at Figure 5, it can be concluded that MPC controller leads to a faster disturbance rejection than PI controller. This is also supported by Figure 6, where reference tracking of a ramp signal from 0 to $80 \mathrm{rad} / \mathrm{s}$ with angular acceleration $\varepsilon_{\text {ref }}=$ $1066.6 \mathrm{rad} / \mathrm{s}^{2}$ is shown. Both figures also show that MPC
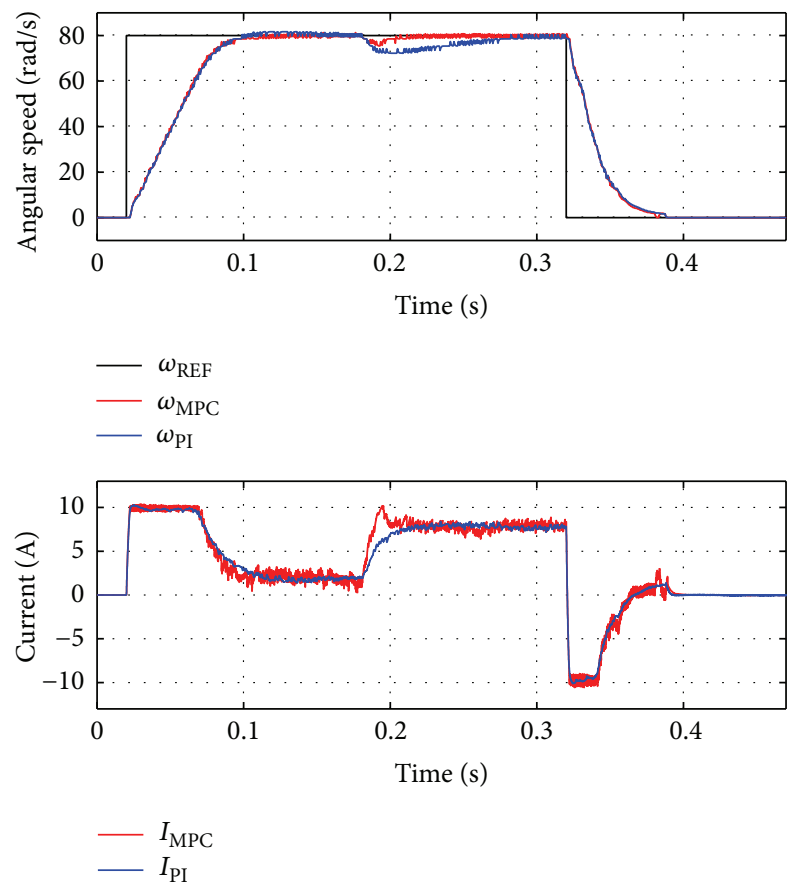

FIGURE 5: Experimental results for the step speed reference. 

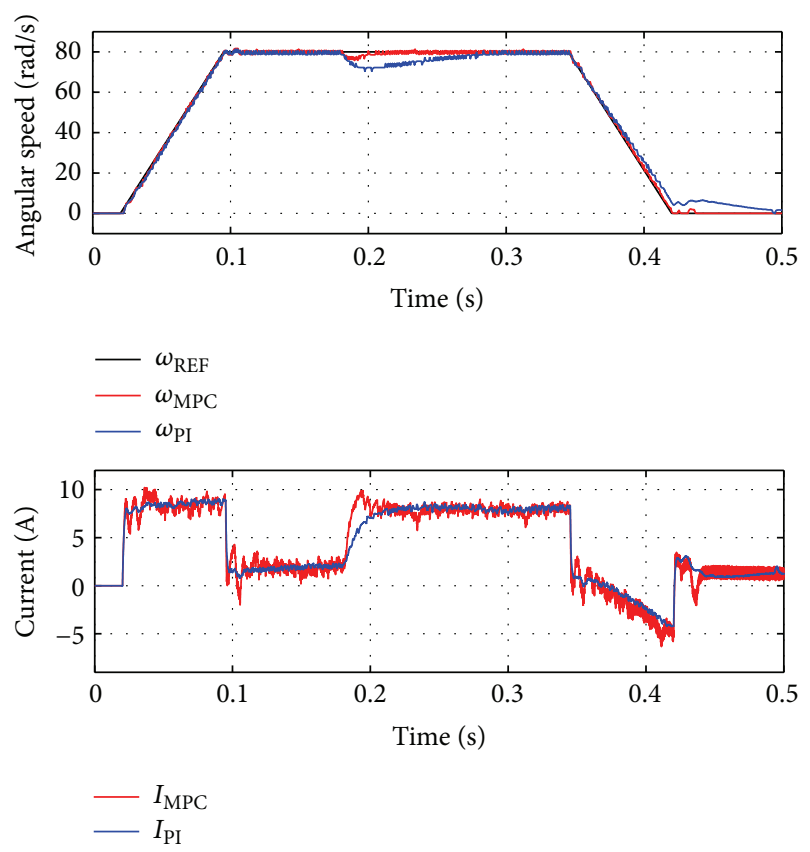

Figure 6: Experimental results for the trapezoidal speed reference and step load changed.

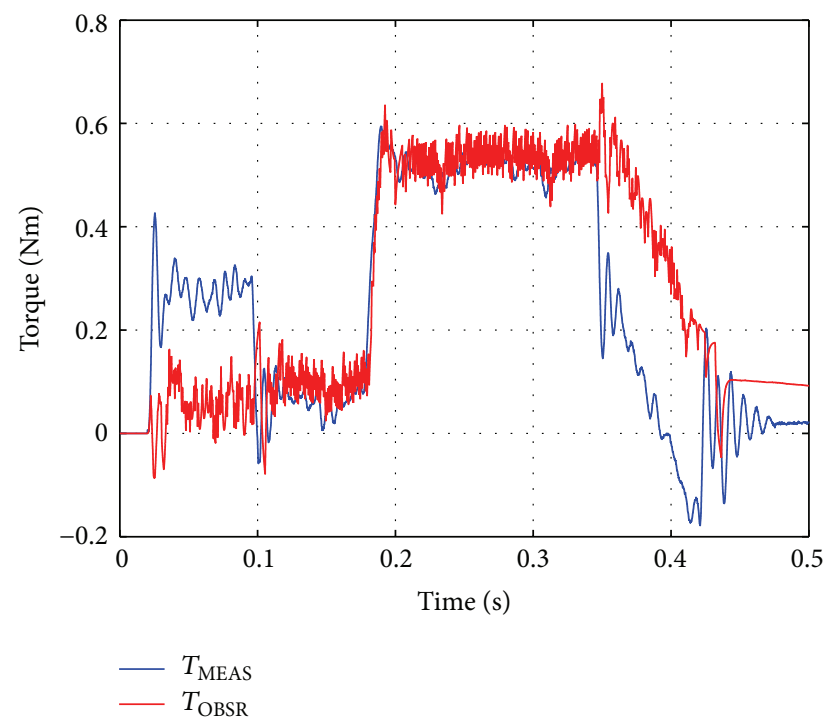

FIGURE 7: Experimental results—torque estimation by Kalman filter.

controller has higher oscillations of machine current than PI controller. These oscillations can be suppressed by increasing of $\lambda_{2}$ in the cost function (28), but this would lead to the deterioration of the speed performance. This is well-known disadvantage of FCS-MPC approaches [12].

The performance of the Kalman filter for load torque estimation is shown in Figure 7. Measured value (blue line) is obtained by the torque transducer. Estimated value (red line) during the acceleration $(t=0.025-0.1 \mathrm{~s})$ does not track the measured value, because the torque sensor also measures a

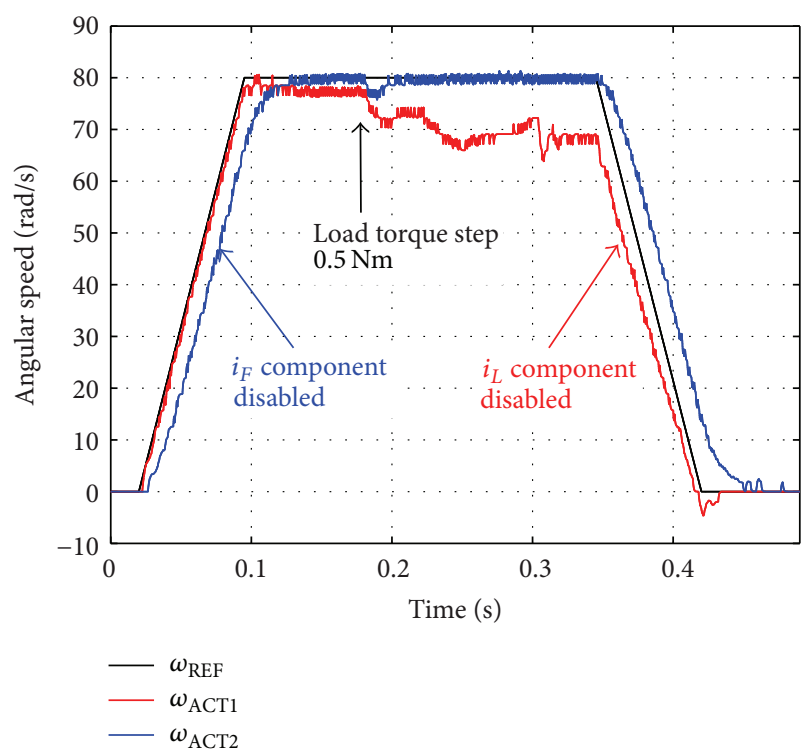

FIGURE 8: Experimental results for the trapezoidal speed referenceinfluence of different current components of the cost function.

dynamic torque, whereas the torque estimator was designed only for estimation of a static torque and load.

Figure 8 shows the influence of the introduced current components on the speed control quality. If the $i_{F}$ current component in the cost function (28) is zero, we can observe considerable dynamic tracking error in the acceleration and deceleration, but the steady-state value of the reference speed is reached. On the other hand, if the $i_{L}$ current component is zero, dynamic tracking of the trapezoidal reference during acceleration is adhered, but, after the loading by external torque, the steady-state accuracy is not preserved. Therefore, a significance of proposed current components $i_{F}$ and $i_{L}$ in the cost function has been proved. We consider this as the main contribution of this paper since these current components can be easily applied to the other types of FCS-MPC controllers, even for AC drives.

\section{Efficiency Issues and Parameter Sensitivity}

8.1. Efficiency Issues. Now we compare number of switching states for both approaches. One transistor turn-on and turnoff yields the $n_{s}=2$ number of switching states. A standard cascade PI controller in Figure 2 uses PWM with the fixed frequency, where the number of switching states (for $\mathrm{H}$ bridge) can be defined as follows:

$$
n_{s}=8 f_{\mathrm{S}} T_{\mathrm{RUN}},
$$

where $f_{S}$ is $\mathrm{PWM}$ carrier frequency and $T_{\mathrm{RUN}}$ is a time period during which the number of switching states is calculated. For example, for $\mathrm{H}$-bridge running with $10 \mathrm{kHz}$ frequency PWM, the overall number of switching states during 1 second is $n_{s}=80000$. On the other hand, MPC controller has a variable switching frequency; thus, exact explicit formula for the number of switching states cannot be performed. 


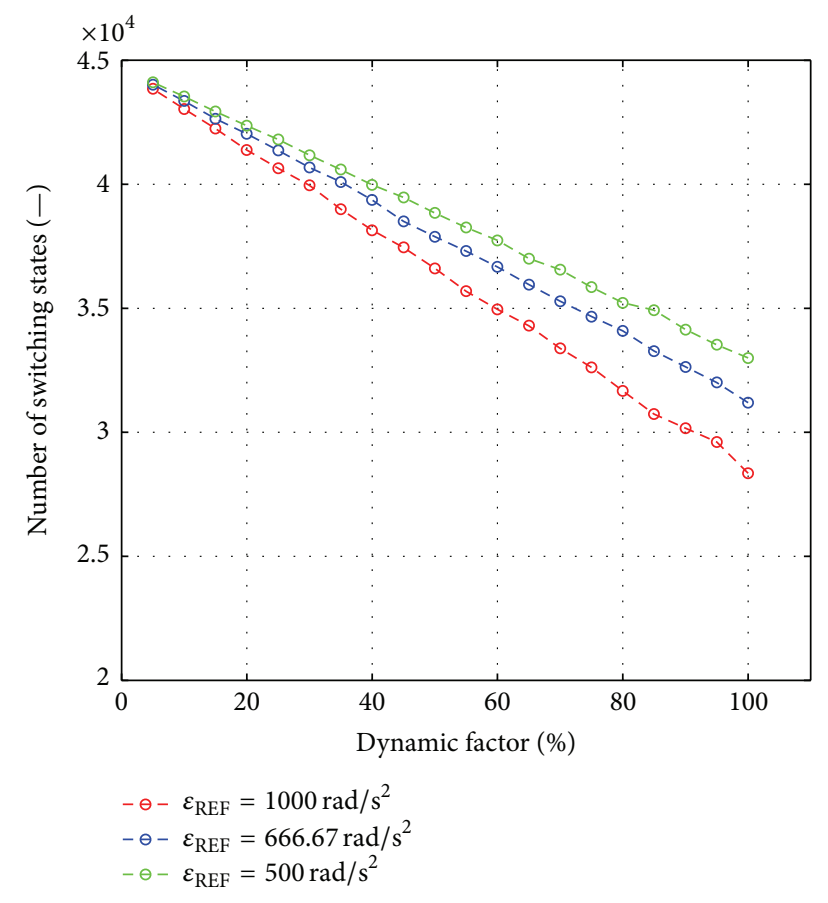

FIGURE 9: Simulation results-number of switching states as a function of the dynamic factor.

It has been found that number of switching states of MPC differs when the drive runs at steady state and transients. Therefore, we proposed a dynamic factor, $d_{f}$, as the following:

$$
d_{f}=\frac{T_{D}}{T_{D}+T_{S T}}=\frac{T_{D}}{T_{\mathrm{RUN}}},[\%],
$$

where $T_{D}$ is a period, where the drive runs at transients (accelerating or decelerating), and $T_{S T}$ is a time, where drive runs at steady state; that is, if $d_{f}=100 \%$, drive is all the time accelerating or decelerating.

The number of switching states for the different values of angular acceleration, $\varepsilon_{\mathrm{REF}}$, obtained by Matlab simulation is shown in Figure 9. It is obvious that, for MPC controlled drive, the number of switching states decreases with increasing dynamic factor. For example, for $d_{f}=100 \%$, the drive needs 28000 switching states, but, for $d_{f}=5 \%$, the drive needs 44000 switching states (the red line). Note that number of switching states was recalculated to the period of 1 second in order to obtain the fairest possible comparison with PIPWM controller.

The number of switching states is directly connected to the switching losses of converter transistors and these losses could present a significant share of the total converter losses and, therefore, the total efficiency of the converter.

For DC-DC converters, the total losses are typically obtained by simulation or by calculation [19] and, in practice, efficiencies of $70 \%$ to $95 \%$ are achieved [20]. In this contribution, an experiment has been provided instead of the calculation. The results are shown in Figure 10. Total efficiency has been measured during the interval of $1 \mathrm{~s}$ with various values of dynamic factor.

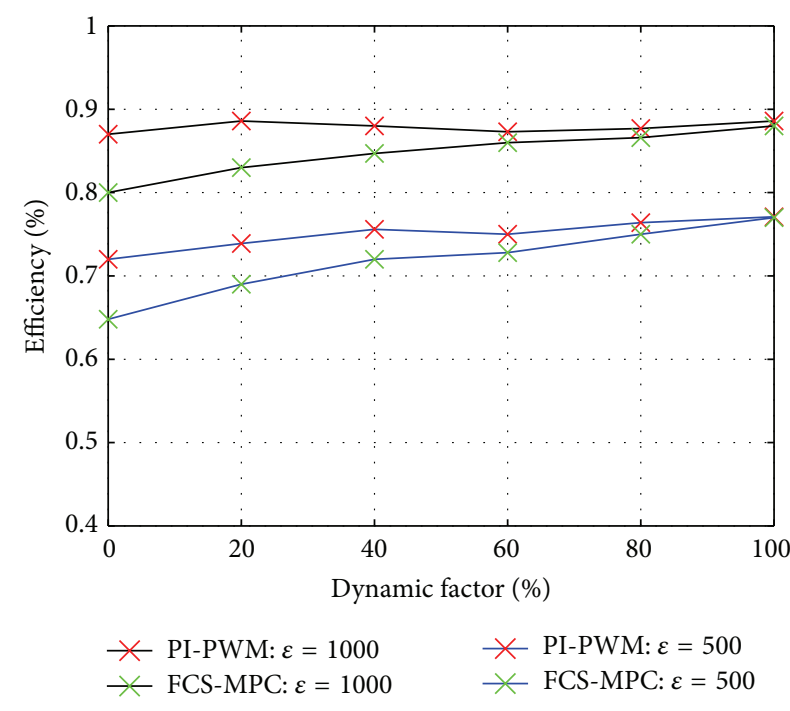

FIGURE 10: Experimental results-measured values of converter efficiency as a function of the dynamic factor.

The input and output voltage and current of the converter have been measured and then the average value of input and output power has been calculated. Note that, for the valid measurement of the output PWM voltage, there is a need for very short sampling time, which is beyond the computational limits of the used simulator; for example, for $1 \%$ precision measurement of $10 \mathrm{kHz}$ output PWM voltage, a voltage sample for calculation has to be stored in memory every $1 \mu \mathrm{s}$. For that reason, PWM voltage frequency in Figure 10 was lowered to $1 \mathrm{kHz}$.

The theoretical maximum of switching frequency using FCS-MPC is $100 \mathrm{kHz}$ (using $10 \mu$ s as sampling time) in steady state. However, during transients, it can be lowered to almost single switching state for a certain time period. Therefore, it can be assumed that, with the higher dynamic factor, the switching losses using FCS-MPC will be lower.

The resulting, experimentally measured efficiency using $1 \mathrm{kHz}$ PI-PWM and FCS-MPC control is depicted in Figure 10. It is obvious that, with lower values of dynamic factor, the efficiency of PI-PWM is better than the efficiency of FCSMPC. On the other hand, with dynamic factor rising to $100 \%$, the performance of both control approaches is comparable. However, it is expected that, with the higher switching frequency of PWM, the switching losses will rise, and so the efficiency of FCS-MPC will overcome the efficiency of PIPWM, even for the lower dynamic factor values.

8.2. Parameters' Variation. In following experiments, the parameters of the FCS-MPC controller remained the same as in Section 7 and real values of inertia and armature resistance have been varied. The variation of the armature resistance, $R_{a}$, for 3 different values of real resistance is shown in Figure 11. It can be observed that, for $180 \%$ change in resistance, speed tracking is not preserved, because the maximum speed error is almost 5\% (green line). In the same case, the current oscillations are mitigated, probably due to 

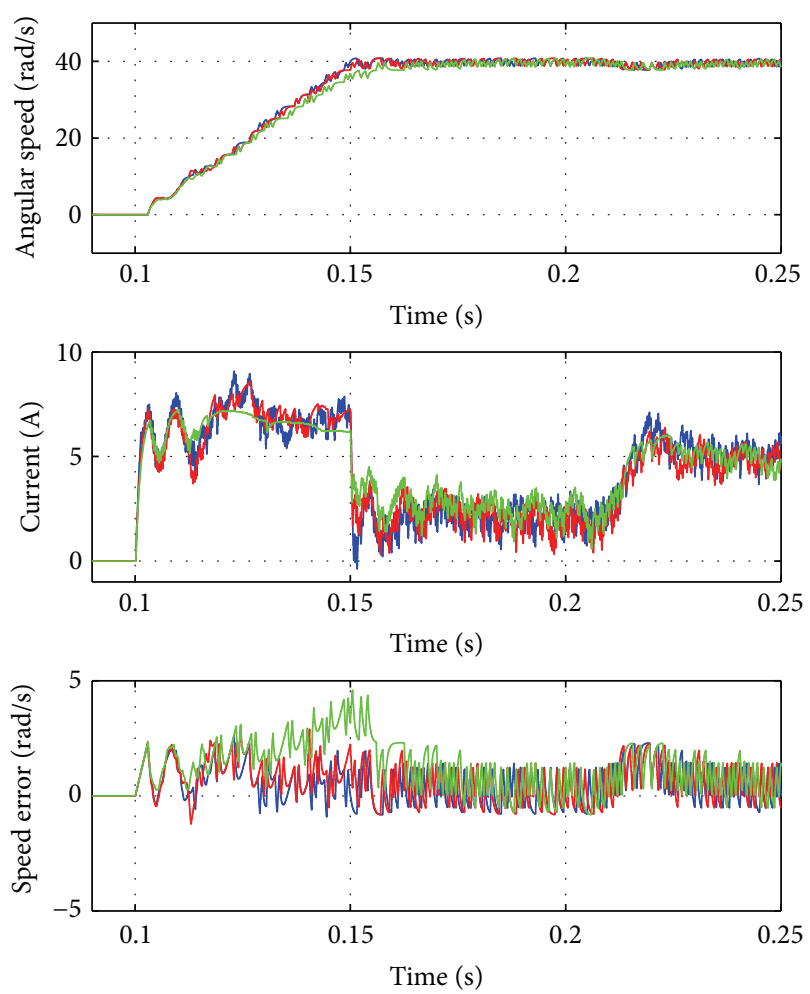

FIGURE 11: Sensitivity to variation of armature resistance, $R_{a}$-blue: $100 \% R_{a}$, red: $150 \% R_{a}$, and green: $180 \% R_{a}$.

the parasitic inductance of the used resistor. Note that the ripple observed in average speed error response is mainly caused by using a low cost encoder with only 1000 ppr, with quantization error, as was shown in Section 3.

Variation of total inertia value is shown in Figure 12. Note that speed reference has been modified in order to keep the current into unsaturated values (i.e., below $10 \mathrm{~A}$ ). The load torque has been applied at $0.6 \mathrm{~s}$. It can be observed that, for the same controller parameters, the speed tracking during acceleration from $t=0.1 \mathrm{~s}$ to 0.4 is preserved for both original and raised inertia value. Even more, the disturbance rejection is excellent and minimal influence of the applied load torque can be seen in the speed error response.

\section{Conclusion}

Finite control set model predictive control has already been used in the control of various electrical drives, but its application for the speed control is still an open problem. An experimental validation of the finite control set model predictive speed control of PMDC machine has been presented.

A new cost function, with the consideration of additional feedforward current components, has been developed. It was shown that the new cost function significantly reduces steady-state error and improves dynamic speed tracking. In addition, proposed MPC controller fluently combines 2level and 3-level voltage control. This controller has been compared with the PI-PWM controller. For comparability, both controllers were tuned to the same settling time and
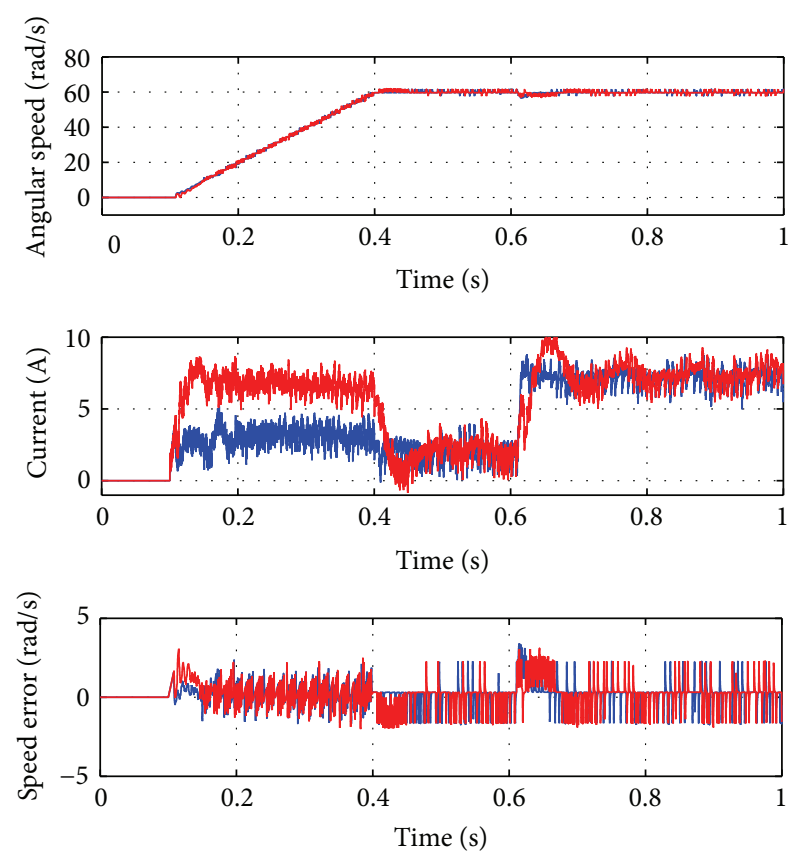

FIGURE 12: Sensitivity to variation of total inertia, $J$-blue: $100 \% J$ and red: $250 \% \mathrm{~J}$.

TABLE 2: PMDC machine parameters.

\begin{tabular}{lcc}
\hline Parameter & Value & Unit \\
\hline$P$ & 250 & $\mathrm{~W}$ \\
$V_{\mathrm{DC}}$ & 12 & $\mathrm{~V}$ \\
$i_{a N}$ & 6 & $\mathrm{~A}$ \\
$i_{\mathrm{MAX}}$ & 10 & $\mathrm{~A}$ \\
$R_{a}$ & 0.6 & $\Omega$ \\
$L_{a}$ & 0.0019 & $\mathrm{H}$ \\
$k_{T}$ & 0.0738 & $\mathrm{Nm} / \mathrm{A}$ \\
$J_{m}$ & 0.000436 & $\mathrm{kgm}^{2}$ \\
\hline
\end{tabular}

overshoot and the measurements were taken under the same boundary conditions.

Presented type of PMDC machine speed control is especially suitable for the drives with very high dynamic demands. It can be concluded that FCS-MPC controller has a fast disturbance rejection and it needs less number of switching states than PI-PWM controller. But its complexity, computational burden, and torque oscillations may be a problem. Finally, it can be confirmed that FCS-MPC controllers are worthy of attention against conventional controllers.

\section{Appendix}

See Table 2.

\section{Competing Interests}

The authors declare that they have no competing interests regarding the publication of this paper. 


\section{Acknowledgments}

This work was supported by the Scientific Grant Agency of the Ministry of Education of the Slovak Republic and Slovak Academy of Sciences (VEGA), under the Project code $1 / 0464 / 15$. This work was also supported by the Slovak Research and Development Agency (APVV), under the Project code APVV-15-0750.

\section{References}

[1] D. Grignion, X. Chen, N. Kar, and H. Qian, "Estimation of load disturbance torque for DC motor drive systems under robustness and sensitivity consideration," IEEE Transactions on Industrial Electronics, vol. 61, no. 2, pp. 930-942, 2014.

[2] M. Tuna, C. B. Fidan, S. Kocabey, and S. Görgülü, "Effective and reliable speed control of permanent magnet DC (PMDC) motor under variable loads," Journal of Electrical Engineering and Technology, vol. 10, no. 5, pp. 2170-2178, 2015.

[3] T. Verstraten, G. Mathijssen, R. Furnémont, B. Vanderborght, and D. Lefeber, "Modeling and design of geared DC motors for energy efficiency: comparison between theory and experiments," Mechatronics, vol. 30, pp. 198-213, 2015.

[4] D. T. Liem, D. Q. Truong, and K. K. Ahn, "A torque estimator using online tuning grey fuzzy PID for applications to torquesensorless control of DC motors," Mechatronics, vol. 26, pp. 4563, 2015.

[5] J. M. Knezevic, "Low-cost low-resolution sensorless positioning of DC motor drives for vehicle auxiliary applications," IEEE Transactions on Vehicular Technology, vol. 62, no. 9, pp. 43284335, 2013.

[6] S. Buechner, V. Schreiber, A. Amthor, C. Ament, and M. Eichhorn, "Nonlinear modeling and identification of a dc-motor with friction and cogging," in Proceedings of the 39th Annual Conference of the IEEE Industrial Electronics Society (IECON '13), pp. 3621-3627, IEEE, Vienna, Austria, November 2013.

[7] M. G. Guerreiro, D. Foito, and A. Cordeiro, "A sensorless PMDC motor speed controller with a logical overcurrent protection," Journal of Power Electronics, vol. 13, no. 3, pp. 381-389, 2013.

[8] J. Rodriguez, M. P. Kazmierkowski, J. R. Espinoza et al., "State of the art of finite control set model predictive control in power electronics," IEEE Transactions on Industrial Informatics, vol. 9, no. 2, pp. 1003-1016, 2013.

[9] J. Rodriguez and P. Cortes, Predictive Control of Power Converters and Electrical Drives, John Wiley \& Sons, 2012.

[10] R. P. Aguilera, P. Lezana, and D. E. Quevedo, "Finite-controlset model predictive control with improved steady-state performance," IEEE Transactions on Industrial Informatics, vol. 9, no. 2, pp. 658-667, 2013.

[11] C. Xia, Y. Wang, and T. Shi, "Implementation of finite-state model predictive control for commutation torque ripple minimization of permanent-magnet brushless DC motor," IEEE Transactions on Industrial Electronics, vol. 60, no. 3, pp. 896905, 2013.

[12] M. Preindl and S. Bolognani, "Model predictive direct speed control with finite control set of PMSM drive systems," IEEE Transactions on Power Electronics, vol. 28, no. 2, pp. 1007-1015, 2013.

[13] E. J. Fuentes, C. A. Silva, and J. I. Yuz, "Predictive speed control of a two-mass system driven by a permanent magnet synchronous motor," IEEE Transactions on Industrial Electronics, vol. 59, no. 7, pp. 2840-2848, 2012.
[14] D. Pavković, J. Deur, and I. Kolmanovsky, "Adaptive Kalman filter-based load torque compensator for improved SI engine idle speed control," IEEE Transactions on Control Systems Technology, vol. 17, no. 1, pp. 98-110, 2009.

[15] C. A. Silva and J. I. Yuz, "On sampled-data models for model predictive control," in Proceedings of the 36th Annual Conference of the IEEE Industrial Electronics Society (IECON '10), pp. 29662971, November 2010.

[16] F. Haugen, Kompendium for Kyb. 2, ved Høgskolen i Oslo, Telemark University College, Department of Electrical Engineering, Information Technology and Cybernetics, Porsgrunn, Norway, 2016, http://techteach.no/fag/seky3322/0708/kalmanfilter/kalmanfilter.pdf.

[17] L. Harnefors, S. E. Saarakkala, and M. Hinkkanen, "Speed control of electrical drives using classical control methods," IEEE Transactions on Industry Applications, vol. 49, no. 2, pp. 889-898, 2013.

[18] J. Vittek, P. Briš, P. Butko, and T. Fedor, "Energy saving position control of PMSM drives with constant, linear and quadratic frictions," in Proceedings of the International Conference on Optimization of Electrical and Electronic Equipment (OPTIM '14), pp. 239-244, Bran, Romania, May 2014.

[19] V. Vavrúš, R. Radvan, and P. Rafajdus, "A power loss calculation method of IGBT SRM converter," in Proceedings of the International Conference on Electrical Drives and Power Electronics (EDPE '15), pp. 521-526, Tatranska Lomnica, Slovakia, September 2015.

[20] R. W. Erickson, DC-DC Power Converters, Wiley Encyclopedia of Electrical and Electronics Engineering, 2007. 


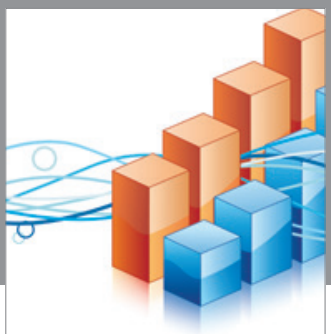

Advances in

Operations Research

vatem alat4

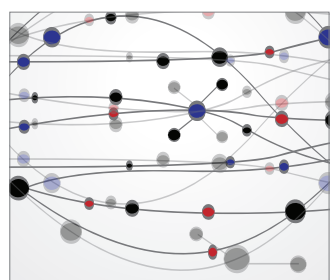

\section{The Scientific} World Journal
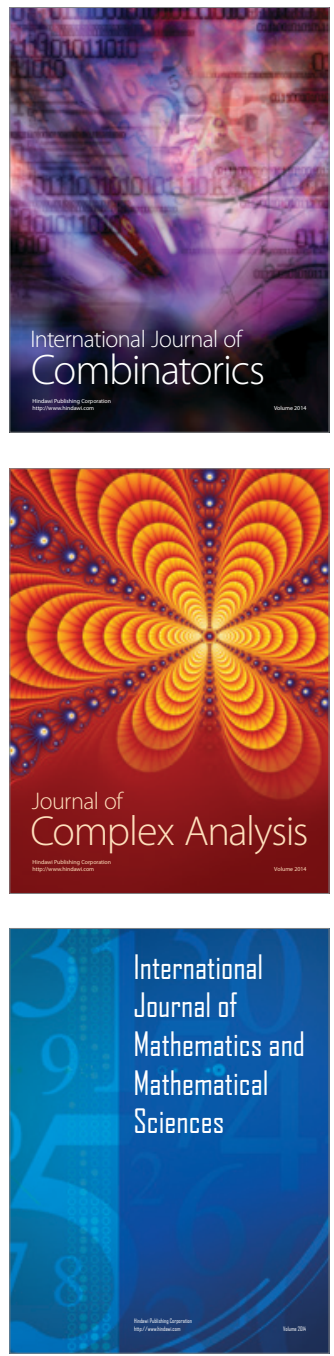
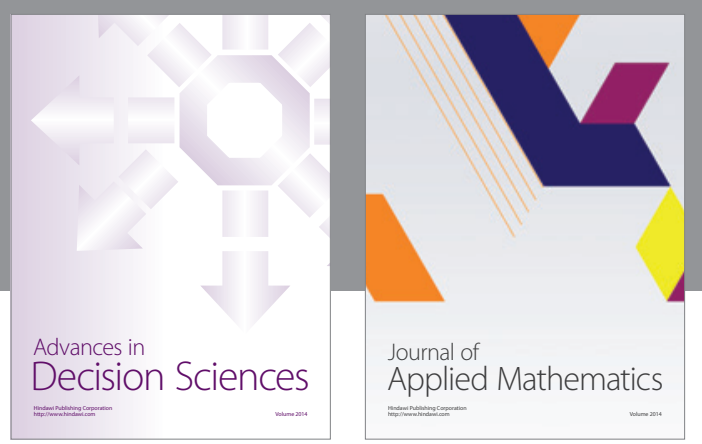

Algebra

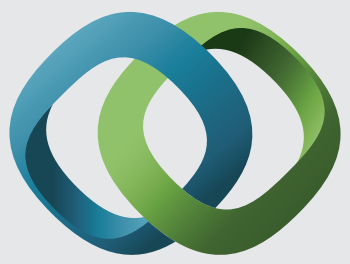

\section{Hindawi}

Submit your manuscripts at

http://www.hindawi.com
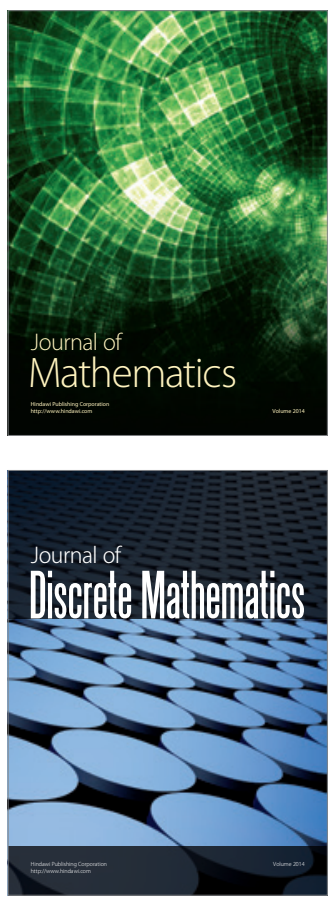

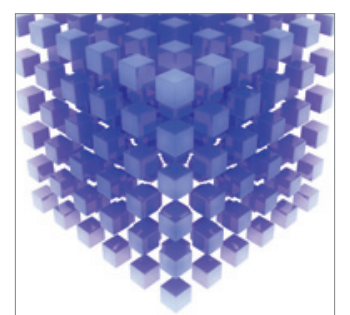

Mathematical Problems in Engineering
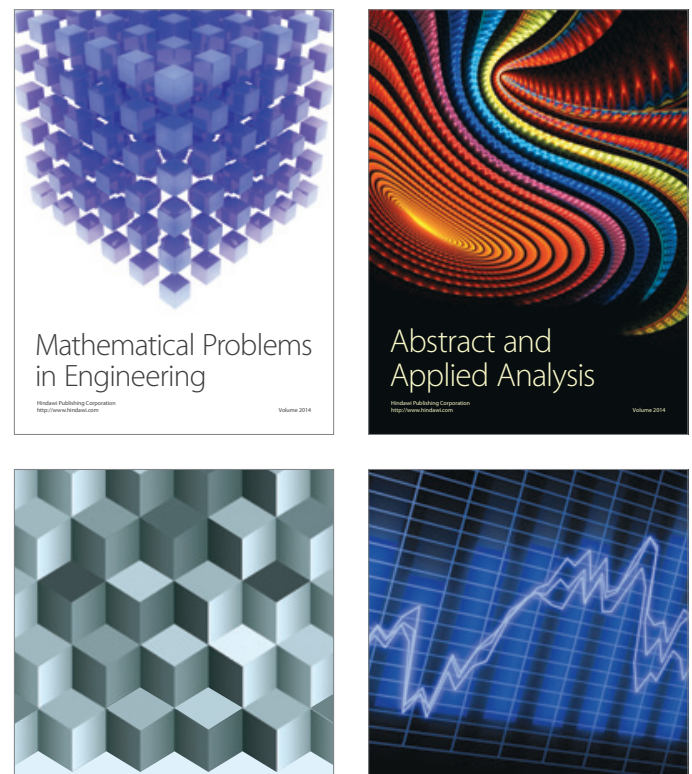

Journal of

Function Spaces

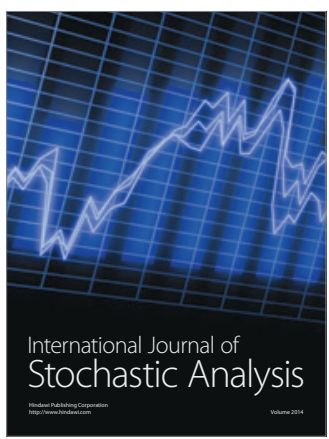

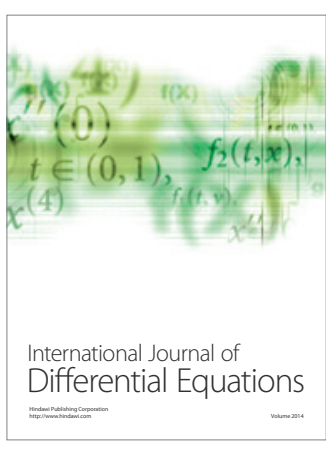
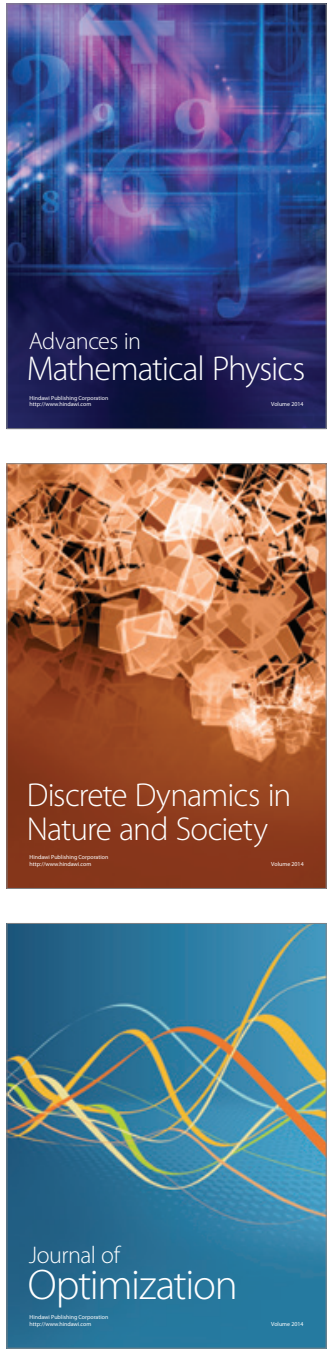